\section{Principles and applications of isotope geology}

Principles of Isotope Geology. By Gunter Faure. Pp. xii +464 . (Wiley: London, Sydney, New York and Toronto, 1977.) $£ 13.60$.

THE late 1940s and early 1950s saw the birth of many ideas and techniques which in the intervening years have grown into the subject of isotope geology. It is a measure of the way in which the subject has developed that this introduction to the principles and applications of isotope geology contains 464 pages, 21 chapters, 2 appendices and a host of literature references. Although the practitioners of isotope geology still make up a relatively small but growing proportion of Earth scientists, the implications of isotope measurements find their way into such a wide variety of geological problems that it is essential for every geologist to have a good awareness of the methods and principles involved if he is to appreciate the power as well as the limitations of the isotopic information to which he may have access.

Dr Faure's book is aimed particularly at the senior Earth sciences undergraduate student entering the field of isotope geology for the first time. I have a feeling also that most practicing geologists would find this a very useful addition to their bookshelves. It is not only a book that can be read systematically from cover to cover but one that can be referred to from time to time when the need arises to refresh the memory as to how some particular isotopic tool 'works' or as to what are the assumptions necessary to draw useful conclusions.

The book is set out in three parts. Chapters $1-5$ are introductory, covering historical aspects, atomic structure, principles of radioactive decay, and mass spectrometry (the basic tool of isotope work). The meat of the book is in the remaining 16 chapters. The many dating methods now in use are described in chapters 6-17, along with examples (from the literature) of their application. The reader meeting the subject for the first time will soon realise that the geochronologist now expects to answer much more than the simple question "How old is this rock?", whatever that means. Rather, he expects, in suitable circumstances, to be able to unravel an often complex history of events, to provide constraints on the rock's origin and mode of formation, and to provide answers to more general questions such as the origin and evolution of the continents and the evolutionary history of the upper mantle.

The last four chapters cover the field of stable isotope studies (hydrogen, oxygen, carbon and sulphur). The applications are too many to summarise in a short review but range over topics as diverse as snow and ice stratigraphy, palaeothermometry, and hydrothermal ore deposits. The author makes no strong demands on the mathematical skills of his readers, and students in particular may find the calculations set out in the text and the many examples following each chapter useful.

This is a very comprehensive book and no doubt specialists in any particular branch of isotope geology may find small points or oversimplifications with which they disagree. For example, the statement

on p167 that interference corrections limit the use of ${ }^{40} \mathrm{Ar}-{ }^{39} \mathrm{Ar}$ dating to samples older than $1 \mathrm{Myr}$ is incorrect. The large field of extraterrestrial isotope studies is hardly mentioned except where it impinges fairly directly on terrestrial applications. However, such criticisms are minor, and this well thought out and well executed introduction to the principles and application of isotope geology should be of use to students and practicing Earth scientists alike.

Grenville Turner

\section{Physicochemical principles and geological systems}

Chemical Petrology. (With Applications to the Terrestrial Planets and Meteorites.) By R. F. Mueller. Pp. 394. (Springer: New York, Heidelberg and Berlin, 1977.) DM 72.80;\$32.10.

CONSIDERABLE advances have been made recently in the application of physicochemical principles to geological systems. This renewal of interest in chemical petrology has stimulated a demand for course texts which both summarise and explain the techniques used and several have been published in the past year. Mueller and Saxena's book is an ambitious attempt to treat the origin and evolution of igneous and metamorphic rocks, related fluids, the terrestrial planets and meteorites from the common viewpoint of the underlying principles of physical chemistry. The book's success in condensing much relevant information will be readily apparent to the reader. As a teaching medium, however, it suffers from its wide scope which results in subjects frequently being dropped before being discussed to a satisfying depth.

The book may be roughly divided into four parts: "Review of Thermodynamics and Kinetics"; "Meteorites and Planetology"; "Metamorphic Petrology"; and "Igneous Petrology". The first part is a review of many of the equations used, including a treatment of the effects of gravity on chemical potential. I found the presentation dry. For the reader without a good working knowledge of thermodynamics (for example, a knowledge of Maxwell's equations), many relationships do not follow obviously, and this may prove a deterrent to many with backgrounds in the Earth Sciences.

Three chapters are devoted to petrogenetics in Space, meteorites, and petrology of the terrestrial planets. These are interesting and informative, and the dis- cussion of condensation reactions is particularly timely. It is regrettable, perhaps, that lunar petrology receives such a limited treatment in comparison with the space allotted to Venus.

The treatment of metamorphism continues the physicochemical paradigm of the book by discussing the origin of microtextures from a thermodynamic viewpoint, and the kinetics of crystal grain growth and of metamorphic reactions. This is followed by a review of experimental studies of the $\mathrm{P}-\mathrm{T}$ stability of various pure mineral assemblages followed by a rather brief analysis of the effects of solid solution. A chapter is devoted to metamorphic mineral facies followed by further discussion of experimental studies of metamorphic systems and a rather disappointing discussion of Schreinemaker's rules.

Igneous petrology is introduced by considering some properties of silicate melts (water solubility density, viscosity, immiscibility). The differentiation of magmas is considered first by analysing diffusion rates in melts and the separation of a fluid phase before discussing the role of crystallisation with a limited use of phase diagrams and variation diagrams. For a book concerned with the physicochemical approach to petrology, it is most surprising that the thermodynamic approach of Carmichael and his colleagues is not mentioned.

This is an interesting, different and stimulating book. Its greatest inadequacy lies in the overemphasis of principles with too few examples and an almost complete lack of worked numerical problems. It is difficult to see its role as a course text especially since the price is likely to prevent most students from purchasing their own copies. It is well produced, although the editing might have been more careful, with some authors' names misspelt throughout the text but correctly in the index and the occasional typographic slip in equations. I am glad, however, to have a copy on my shelf.

D. G. Fraser

D. G. Fraser is Lecturer in Geochemistry at the University of Oxford, UK. 